\title{
Evolution of Boolean Networks: Contingency Versus Necessity in Biology
}

\author{
Stephen Gilmore*,1 and David Green ${ }^{2}$
}

\author{
${ }^{1}$ Dermatology Research Centre, School of Medicine, University of Queensland, Princess Alexandra Hospital, Brisbane, \\ 4102, Australia \\ ${ }^{2}$ Information Technology Services, University of Queensland, Brisbane, 4072, Australia
}

\begin{abstract}
Many scientists are convinced that the Modern Synthesis - the current paradigm in evolutionary biology needs to be expanded within the framework of an Extended Evolutionary Synthesis (EES). A major task for the EES is to provide an explanation for the origins and extant features of biological complexity. Here we address this issue, focusing our investigation on genetic network architectures, motifs, and dynamical behaviour, by developing an intuitive and essentially parameter-free evolutionary model of transcriptional regulation where the self-replicating digital organisms are Boolean networks, and where fitness is determined by their information-processing capacities. We validate our choice of fitness function by demonstrating that our evolved networks exhibit typical biological features of extant genetic regulatory networks: sparse connectivity, scale-free out-degree (within our range of measurement) and exponentially decaying indegree distributions, significant clustering, a high proportion of feed-forward loop (FFL) network motifs, a prominence of canalising logic at the promoter, plasticity, and distributed robustness to mutation. In addition, the dynamics of our evolved networks feature simple attractor cycles that are robust to perturbations and exhibit self-organised criticality. In networks evolved without gene duplications, we show that the key architectural signatures noted above are absent. Surprisingly, the canalising fraction is much higher in comparison with networks evolved with gene duplications. These results suggest network properties of extant gene networks require gene duplications in order to evolve, and that these properties undergo positive selection, where they contribute to the global stability of the networks. By demonstrating that networks evolved without gene duplications are robust and, like their scale-free counterparts, also exhibit self-organised criticality, this work highlights the interplay between contingent mechanism, such as gene duplications, and selection, in determining evolutionary outcomes.
\end{abstract}

Keywords: Boolean networks, genetic networks, scale-free, robustness, evolvability.

\section{INTRODUCTION}

A major challenge for evolutionary theory is to explain how and why organisms develop complex features as a consequence of selection, mutation, and other non-selective forces $[1,2,3]$. Although the reconstruction of phylogenies by integrating information from palaeontology, 'evo-devo' and modern systems biology has met with partial success [4,5], efforts to trace population and molecular events in detail are undermined by a lack of historical data. Moreover, attempts to determine which features of biological systems perform vital roles, and which are incidental to physical or evolutionary mechanisms, are confounded by the singular origin of all extant life on Earth.

To be sure, the latter uncertainty is exemplified by considering the architectural signatures associated with genetic regulatory networks: why, for example, are out-degree (see Table $\mathbf{1}$ for a glossary of terms) distributions scale-free? Are these architectures performing an indispensable biologic function, or are they simply a relic secondary to the mecha-

\footnotetext{
*Address correspondence to this author at the Dermatology Research Centre, University of Queensland, School of Medicine, Princess Alexandra Hospital, Brisbane, Queensland, 4102, Australia;

Tel: + 6173240 5467; Fax: + 6173240 5663;

E-mail: s.gilmore1@uq.edu.au
}

nisms of genome expansion? While some authors have suggested such architectures confer biologic advantage, such as robustness or efficient information transfer $[6,7,8]$, and therefore may be selected for, others have offered more pedestrian explanations: for example, it has been suggested that large-scale network structures can arise during neutral evolution [9], or can develop passively as a consequence of genome expansion via gene duplications [10,11,12]. Evolutionary modelling has also been invoked: previous work has shown that the development of scale-free architectures, perhaps not surprisingly, can develop in evolutionary runs associated with preferential attachment mutations $[6,13,14]$. At the intermediate level of gene network organisation, the preponderance of specific network motifs, such as the feedforward loop motif [15], also demand explanation. While some authors have suggested they arise locally and in response to selective forces [16], others have argued, analogously to the argument associated with the development of scale-free degree distributions, that the frequency of such motifs can be explained by the mechanisms of genome expansion [17].

Previous work has shown many genes are regulated at the promoter by transcription factors in a combinatorial manner $[16,18]$, and the logic has been shown to belong to a class of functions that possess the canalising property [18]. What is the origin and purpose of this class of combinatorial logic in 
Table 1. Glossary of terms

\begin{tabular}{|c|c|}
\hline Cellular Automata & $\begin{array}{l}\text { A grid of cells, connected to a variable number of } \\
\text { nearest neighbours, where each cell can exist in } \\
\text { two or more states, and where the state of each cell } \\
\text { is updated as a function of the set of states of its } \\
\text { connected nearest neighbours. }\end{array}$ \\
\hline $\begin{array}{l}\text { Random Boolean } \\
\text { Network (RBN) }\end{array}$ & $\begin{array}{l}\text { A generalised cellular automata where the archi- } \\
\text { tecture of connections between cells is random, } \\
\text { and where the updating Boolean rules for each cell } \\
\text { are chosen at random. }\end{array}$ \\
\hline Connectivity & $\begin{array}{l}\text { The density of connections between cells in a } \\
\text { RBN, usually defined as the number of connec- } \\
\text { tions divided by the number of possible connec- } \\
\text { tions. }\end{array}$ \\
\hline In-degree & $\begin{array}{l}\text { The pattern of connectivity of an RBN - its archi- } \\
\text { tecture - is usually represented as a directed } \\
\text { graph; that is, the output of one cell is input to } \\
\text { another cell, not necessarily vice versa. The in- } \\
\text { degree of a cell is its number of input connections. }\end{array}$ \\
\hline Out-degree & $\begin{array}{l}\text { The number of output connections from a given } \\
\text { cell. }\end{array}$ \\
\hline $\begin{array}{l}\text { Clustering Coeffi- } \\
\text { cient }\end{array}$ & $\begin{array}{l}\text { The likelihood that two connected cells are both } \\
\text { connected to a third cell, averaged over all con- } \\
\text { nected pairs. }\end{array}$ \\
\hline Canalising Rules & $\begin{array}{l}\text { A type of Boolean rule where the state of a cell is } \\
\text { determined by the input from only one or more } \\
\text { input cells, regardless of the states of the other } \\
\text { input cells. }\end{array}$ \\
\hline Attractors & $\begin{array}{l}\text { Patterns of network states in an RBN that can be } \\
\text { unchanging (fixed point), recurrent (oscillatory) or } \\
\text { non-repeating (chaotic). }\end{array}$ \\
\hline Avalanche Size & $\begin{array}{l}\text { The number of iterations required for a RBN to } \\
\text { reach an attractor following a single cell perturba- } \\
\text { tion to an RBN settled on an existing attractor. }\end{array}$ \\
\hline $\begin{array}{l}\text { Self-organised } \\
\text { Criticality }\end{array}$ & $\begin{array}{l}\text { A system where the dynamics evolve toward a } \\
\text { state where the distribution of avalanche sizes } \\
\text { follows a power law. }\end{array}$ \\
\hline $\begin{array}{l}\text { Mutational Ro- } \\
\text { bustness }\end{array}$ & $\begin{array}{l}\text { Fraction of non-viable offspring following random } \\
\text { mutations; to reduce statistical error the robustness } \\
\text { of a given network is calculated following } 1000 \\
\text { independent random mutations. }\end{array}$ \\
\hline Evolvability & $\begin{array}{l}\text { Fraction of offspring with increased fitness fol- } \\
\text { lowing random mutations; to reduce statistical } \\
\text { error the evolvability of a given network is calcu- } \\
\text { lated following } 1000 \text { independent random muta- } \\
\text { tions. }\end{array}$ \\
\hline Plasticity & $\begin{array}{l}\text { Fraction of offspring with either no change or } \\
\text { increased fitness following rewiring mutations. }\end{array}$ \\
\hline
\end{tabular}

extant biological networks? Do they exist, as suggested by Kauffman [19], to sustain global ordering of the networks' behaviour, or, can their increased frequencies be attributed to additional or alternative explanations: for example, to facili- tate mutational robustness? From the dynamical perspective, it has been shown by a number of investigators that the behavioural responses of genetic regulatory networks are critical: perturbations to gene expression lead to cascades of gene expression changes where the magnitudes of those changes follow a power law [20]. While it may seem likely that dynamic behaviour of this kind has evolved to facilitate robust yet flexible signalling responses, the origins of such cellular responses are less clear. An important question thus arises: are the scale-free architectures found in biological networks a necessary condition for critical dynamics to evolve?

Taken together, the examples above highlight an unresolved problem with regard to the structure and behaviour of biological networks: contingency versus necessity. In the following sections we develop an evolutionary model with the aim of gaining insight into this problem, paying attention to the development of architectural signatures, dynamical behaviour, robustness, and evolvability. We are motivated by the utility of evolutionary modelling: indeed, it is well recognised that a complementary approach to the investigation of life's evolutionary history is to study the evolution of digital organisms or in-silico genetic networks; the advantages of these methodologies have been described elsewhere $[21,22]$.

Following Kauffman [19] and a large number of subsequent investigators, we model genetic regulatory networks with Boolean networks. A model genetic regulatory network based on Boolean logic is a type of generalised cellular automata, where the genes, represented by vertices in a directed graph, can be either in on or off states, corresponding to expression or repression of those genes. Gene states are updated simultaneously and discretely. Regulation at the promoter is modelled using Boolean logic: the state of vertex $V_{A}$ at time $\mathrm{T}=t+1$ is determined by the state(s) of its input vertices $\left(V_{B}, V_{C}, V_{D}, \ldots\right)$ at time $\mathrm{T}=t$, calculated using a fixed Boolean rule associated with vertex $A$. For example, a simple Boolean rule associated with gene $A$ with inputs from genes $B$ and $C$ might be as follows: if any or both of the input genes $B$ and $C$ are expressed at T $=t$, then gene $A$ is expressed at time $\mathrm{T}=t+1$, otherwise gene $A$ is repressed at time $\mathrm{T}=t+1$. Fig. (1a) shows a five-vertex network, while Fig. (1b) shows a pattern of gene expression associated with this network. In Fig. (1b) time progresses down the page, and each gene is either expressed (yellow) or repressed (purple). Note that the state of the network at any time is represented by a horizontal row, and that this state repeats after $t$ $=4$ updates. The repeating pattern of network states forms an attractor of the network. In general, there can be many attractors associated with a given network (where the latter is defined by its architecture and list of associated Boolean rules). The basin of attraction of a given attractor is the set of states of the network that will evolve toward that attractor and stay there. Basins of attraction are not usually of equal size; from a random initial state, a network is more likely to evolve to particular attractors.

A Boolean network is then considered an 'agent'; to model the evolution of a population we track the changes in a cohort of networks over time. Briefly, each network among a population is assigned a fitness value based on its attractor pattern; at each replication round, individual networks are chosen randomly with a frequency proportional to fitness. 


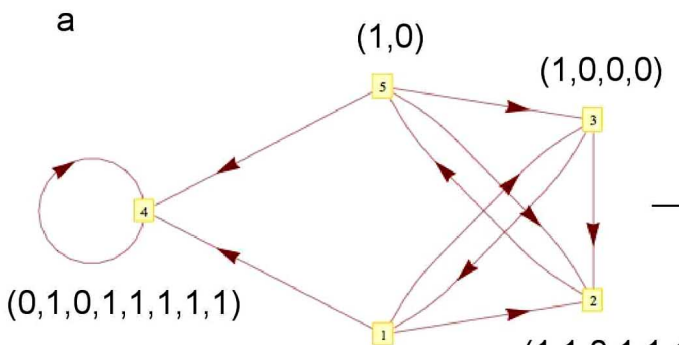

$(0,1)$

$(1,1,0,1,1,1,1,1)$

C

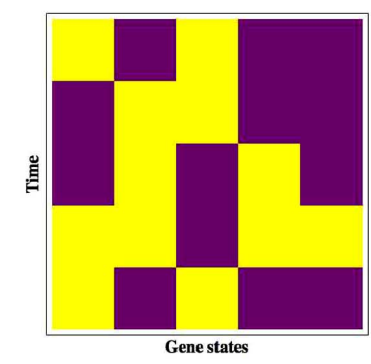

b

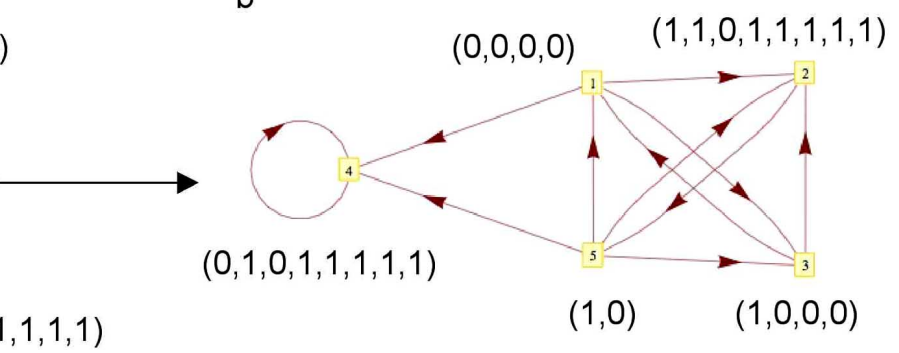

d

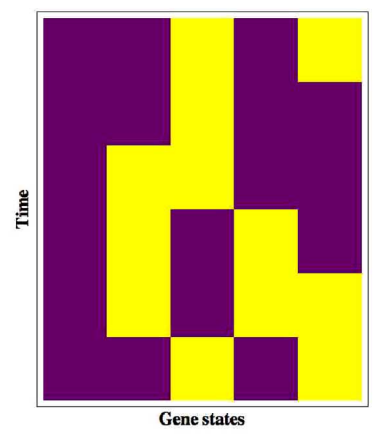

Fig. (1). The Boolean representation of transcriptional regulatory networks. Shown is a five vertex network representing a simple five gene regulatory network (a). Note that the graph is directed, indicating transcription factors derived from one gene act in one direction on the promoter regions of other genes, controlling their output via Boolean logic. Each vertex is then associated with a Boolean rule that determines the on-off status of the vertex at the next time step as a function of the status of the in-coming connected vertices. For example, in (a) vertex 5 has only one input; since there are four potential rules in this case (on / on; on / off; off / on and off / off ) for each respective on / off input, the Boolean rule specifies a choice between one of these four alternatives. Accordingly, it is $2^{L}=2$ bits long, where $L$ is the number of incoming edges. An attractor pattern associated with network (a) is shown (b), where a yellow square represents an 'off' gene and a purple square represents an 'on' gene. The mutual information associated wth this attractor pattern is then used to calculate the fitness of (a). When a mutation occurs (see Table 1) a different network will be generated (c). In this case a mutation at the promoter of vertex 1 has led to a new binding site for the product of vertex 5 , leading to a new directed edge from vertex 5 to vertex 1 . Note the Boolean rule associated with vertex 1 needs to change to accommodate the new edge; it is now 4 bits long and is chosen at random. Network (c) now has a new attractor (d), determined at its inception from a random initial state, which, in this case, is longer than (b). Even though the mutual information measure of (d) may be greater than (b), according to our $90 \%$ attractor homology rule, the fidelity of reproduction fails here; the mutation to (a) has produced non-viable offspring (c).

The chosen network is then duplicated and replaces another network from the population at random. The duplicated network may remain identical to its parent, or, with a certain probability, mutate. A mutation can affect the offspring by a change to the Boolean logic at a single vertex, or it may affect the offspring by a change to the wiring pattern associated one or more vertices (see Table 2 and Fig. 1). The important steps in calculating the fitness of a network are twofold: first, defining an attractor pattern associated with the network; and second, the methodology that calculates fitness based on that attractor pattern. We define the attractor pattern associated with a given network as the attractor pattern associated with a random choice of initial state, calculated immediately after the new network is generated by the reproduction process. We denote this attractor as a network's functional attractor ${ }^{1}$. While this procedure may occasionally lead to an attractor pattern associated with a small basin of attraction, over evolutionary time, it will select for attractors associated with large basins of attraction. Finally, to calculate fitness, we use a measure from information theory -

\footnotetext{
${ }^{1}$ These attractor patterns must be reached with high probability following mutations in order for its associated network to remain viable, hence they will, for a successful species, be associated with disproportionately large basins of attraction.
}

mutual information - which, in a non-technical sense, can be considered the loss of uncertainty about the expression pattern of gene $B$ when the expression pattern of gene $A$ is known (or vice-versa). As we discuss in more detail in the Methods, the mutual information between two gene expression patterns is a measure of both the complexity of the patterns and the correlation between patterns. To calculate the fitness of the network we simply sum the mutual information of all gene pairs: calculating the fitness of the five-vertex attractor pattern shown in Fig. (1b) thus involves summing the mutual information of $N(N-1) / 2$ or $(5 \times 4) / 2=10$ gene pairs (Fig. 2).

Here we identify our networks or 'agents' as network modules - relatively isolated groups of interconnected genes and transcription factors that are generally associated with a particular function [15]. There exist a number of reasons why our modelling approach is fundamentally different from the large body of work that preceeds it: first, it is associated with an unbiased ${ }^{2}$ mutation look-up table (Table 2); second, the use of mutual information as a fitness measure facilitates

\footnotetext{
${ }^{2}$ An unbiased mutation look-up table keeps the same probabilities for gene additions or deletions, keeps the same probabilities for the removal or addition of single links between genes, and does not invoke preferential attachment or deletion.
} 


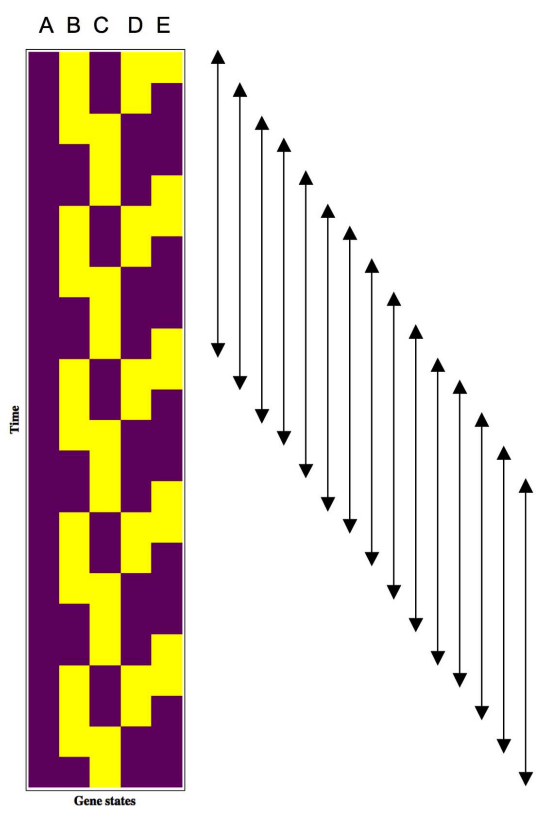

Fig. (2). Calculating the fitness of a network from its attractor pattern. In this example, in contrast to the 256 sequential states sampled in the simulations, we restrict the number of consecutive states to 24 for simplicity. We consider a five gene network. To begin, we calculate the mutual information between genes $\mathbf{A}$ and $\mathbf{B}$, by calculating the entropy $H_{A}$ of $\mathrm{A}$ and the entropy $H_{B}$ of $\mathrm{B}$ (using ten iterations of the network dynamics - this then corresponds to the fifteen time series patterns shown by the arrowed lines). WE then calculate the joint entropy $H_{A B}$ of $\mathbf{A}$ and $\mathbf{B}$ (as before, by using the fifteen time series patterns corresponding to the arrowed lines). The mutual information between genes $\mathbf{A}$ and $\mathbf{B}$ is then $H_{A}+H_{B}-$ $H_{A B}$. We then repeat this calculation for all gene pairs (AB, $A C$, $\mathrm{AD}, \mathrm{AE}, \mathrm{BC}, \mathrm{BD}, \mathrm{BE}, \mathrm{CD}, \mathrm{CE}, \mathrm{DE})$. The fitness of the network is then given by the sum of all mutual information gene pair values.

relatively unconstrained evolution by avoiding teleology ${ }^{3}$; and third, although we employ the abstract nature of information as a measure of fitness, we combine this with the high semantic value of our networks' attractor states, the latter of which is essentially coded environmental information.

Here we validate our choice of fitness function by showing that many of the small and large-scale architectural and dynamic features of extant genetic regulatory networks can be reproduced in a simple and unbiased evolutionary model comprising mutation, selection and reproduction. In the sections that follow we introduce the term contingent mechanism and show why gene duplications are a prime example. We demonstrate that, within the context of our model, while gene duplications are required to generate extant biological network features, outcomes could have been different - similarly performing networks can evolve without duplications or scale-free architectures. Finally, we discuss the implications of our findings with respect to network robustness and evolvability.

\footnotetext{
${ }^{3}$ We suggest that any evolutionary model that incorporates teleology is somewhat flawed since natural systems do not evolve toward some future pre-determined goal; they can only respond to the present.
}

\section{METHODS}

\section{Boolean Networks}

Our digital organisms are self-replicating Boolean networks that compete for computer memory among a 250 network population. We model the transcriptional network using the representation $G=\left(V_{N}: E_{M}\right)$ where $G$ is a directed graph containing $N$ vertices and $M$ edges. Each vertex $V_{i}$ with $L_{i}$ input edges is associated with a binary vector of length $2^{L}$ corresponding to a Boolean rule that determines the status of the vertex at the next step as a function of the status of its incoming connected vertices (Fig. 1). Although the Boolean approach to modelling transcriptional regulation has limitations, such as its simplistic on-off representation of gene activity, or its inability to accommodate non-Boolean logic at the promoter, it has many advantages; the most important is that it allows for the modelling of global behaviour when there are many interconnected elements [19].

\section{Fitness Function}

The existence of modular functions, such as morphogenetic events that can be tweaked without affecting the whole organism [23], allow us to justify imposing a suitable fitness function on our evolving population. We define our fitness function $f$ as the sum of mutual information (MI) between all pairs of vertices on the network, given by

$$
f=\sum_{i} \sum_{j} H_{i}+H_{j}-H_{i, j}
$$

where the entropies $H$ are calculated using the frequencies of the individual and joint vertices' 10 -state sequential temporal patterns, sampled over 256 iterations of the networks' functional attractor (Fig. 2). This multi-information measure [24] thus assigns fitness as a measure of functional complexity which we identify as isomorphic with coupling and coordination in gene expression [25]. Finally, we assume that a network with more vertices has the potential for more information processing.

We justify our fitness function on several grounds. First, the evolution of multi-cellularity, and presumably fitness gains, has involved an expansion in the information processing of developmental regulatory modules: for example, the intercalation of regulatory elements within a simpler system has underpinned the evolution of the metazoans' developmental control kernels [26]. Second, increased diversity of an organism's physiologic capabilities, such as the sensory transduction repertoire of bacteria living in complex environments, facilitates reproductive success and requires greater regulatory sophistication [27]. Third, and as briefly noted in the Introduction, in contrast to other evolutionary models, where the fitness of digital organisms is equated with their ability to perform specific information processing tasks [21], or pattern-matching [28,29,30], we do not impose a specific evolutionary goal on the population; rather, the environment is defined implicitly by the particular attractor pattern exhibited by our networks. In other words, while the semantic value of the particular pattern exhibited by a network's functional attractor is high, the population of networks are not evolving toward some pre-determined 'fitter' pattern. Analogous to the ideas introduced by Adami [31] we 
Table 2. Mutation-type look up table. Note how we have paid careful attention to the interplay between the types of mutations and their effects on the networks' architecture and logic. For example, while a mutation to a promotor will lead to a new Boolean rule at that promoter, a mutation to a transcription factor's gene will not lead to new logic at its target promoter; in such cases although the deletion of the corresponding edge requires a new bit length for the rule at that target promoter, is must be identical to the previous rule assuming absense of the lost transcription factor

\begin{tabular}{|c|c|c|c|}
\hline \multirow{2}{*}{ Mutation Type } & \multicolumn{2}{|c|}{ Network Change } & \multirow{2}{*}{ Probability } \\
\hline & Architectural Change & Rule Change & \\
\hline \multirow[t]{4}{*}{ Promoter mutation } & No change & New random rule & 0.40 \\
\hline & New edge & New random rule & 0.175 \\
\hline & Deletion of edge & New random rule & 0.0875 \\
\hline & Loss of all incoming edges & New random rule & 0.075 \\
\hline \multirow[t]{2}{*}{ Transcription factor mutation } & Deletion of edge & $\begin{array}{l}\text { Same rule as per pre-mutated transcrip- } \\
\text { tion factor absent }\end{array}$ & 0.0875 \\
\hline & Loss of all outgoing edges & $\begin{array}{l}\text { Same rules as per pre-mutated transcrip- } \\
\text { tion factor absent }\end{array}$ & 0.075 \\
\hline \multirow[t]{2}{*}{ Duplication } & $\begin{array}{l}\text { All outgoing edges as per duplicated vertex. } \\
\text { New random incoming edge }\end{array}$ & $\begin{array}{l}\text { Same rules for outgoing edges. New } \\
\text { random rule for incoming edge }\end{array}$ & 0.02 \\
\hline & $\begin{array}{l}\text { All incoming edges as per duplicated ver- } \\
\text { tex. New random outgoing edge }\end{array}$ & $\begin{array}{l}\text { Same rules for incoming edges. New } \\
\text { random rule for outgoing edge }\end{array}$ & 0.02 \\
\hline $\begin{array}{l}\text { Horizontal t/fer } \\
\text { a. External promoter mutation } \\
\text { b. External transcription factor } \\
\text { mutation }\end{array}$ & $\begin{array}{l}a \text {. New ingoing edge to new vertex } \\
b \text {. New outgoing edge from new vertex }\end{array}$ & $\begin{array}{l}a \text {. New random rule } \\
b \text {. Same rule as per new transcription } \\
\text { factor absent. New random rule for new } \\
\text { vertex }\end{array}$ & $\begin{array}{l}\text { a. } 0.005 \\
\text { b. } 0.005\end{array}$ \\
\hline Deletion & Loss of all incoming and outgoing edges & $\begin{array}{l}\text { Same rules as per deleted transcription } \\
\text { factor absent }\end{array}$ & 0.05 \\
\hline
\end{tabular}

interpret the sequence of specific states of the networks' functional attractors as coded environmental information. Since we do not simulate a single-goal optimisation process, we capture the essence of co-evolution and hence avoid the problem of coupling evolution and teleology. Finally, our fitness function has the realistic and desirable property of not corresponding to a well-defined metric in genome space.

\section{Monte Carlo Updating Scheme}

At each replication round, a network is chosen at random with a frequency proportional to fitness and replicates. The daughter network mutates with probability $p=m N$ / $(1+m N)^{4}$ where $m$ is the mutational burden and $N$ is the vertex count. The types of mutations that can occur and their associated probabilities are shown in Table 2; although these rules and their frequencies are chosen heuristically, they are simple, biologically relevant [15], and, as noted above, unbiased.

If a mutation occurs, the daughter network's first attractor to be reached from random initial conditions is used to calculate its fitness, thus, as noted in the Introduction, we implicitly select for networks with highly non-uniform sized basins of attraction. To remain viable, the daughter network

${ }^{4}$ This simple and monotonically increasing relation ensures $p<1$ for any $m$ and $N$. is constrained to share greater than $90 \%$ attractor homology with its parent since gene expression patterns need to be similar otherwise they will not function as before. We apply our $90 \%$ attractor homology rule as follows: an attractor pattern can be written as a matrix of dimensions $N \times 256$, where $N$ are the number of vertices and 256 the number of sequential network states sampled. When comparing attractors between the old network and the newly mutated network, we find an alignment between the two matrices that maximises the number of correct matches at all positions. The fraction of matching positions is then is a measure of attractor homology. Finally, the new network's attractor length is required to be less than 256 states. Fig. (1) shows an example of this process for a simple network of size $N=5$. Irrespective of whether a mutation occurs, the daughter network then replaces another network within the population at random. If the mutated network fails to satisfy our constraints it is rendered non-viable and assumes a very low fitness. The constraints on viability ensure that the particular attractor patterns exhibited by our networks - which, as we noted above, are meaningful in the context of their environments - are preserved with high fidelity, and serve to exclude long period attractors, which could potentially include chaotic attractors, as biologically viable. Indeed, both long and shorter length chaotic attractors are not expected to evolve since the likelihood of a chaotic network mutating from either a cha- 
otic or non-chaotic network with $90 \%$ or greater attractor homology is essentially zero. For the seed population where $N=4$, calculating fitness by sampling over 256 iterations of its functional attractor ensures chaotic behaviour is associated with fitness values close or equal to zero.

\section{Simulations}

We begin all simulations with a seed population of 250 randomly generated 4-vertex networks and calculate the fitness for each. Although this procedure allows different networks and attractors to be viable, this variation is small compared with the space of all possible 4-vertex networks. For networks that grow from 4 to 50 vertices, our mutation probability expression $p$ with $m=0.0025$ yields a probability of mutation per replication event ranging from $p=0.01$ to $p=$ 0.11 , consistent with a network module embedded within a primitive genome of 400 genes operating near its error threshold $^{5}$ [32]. We therefore take $m=0.0025$ as our baseline mutation load, and terminate our simulations when any member of the evolving population reaches $N=50$. To begin, we run 28 independent and randomly seeded evolutionary histories from $N=4$ to $N=50$ at $m=0.0025$. To investigate whether evolved network properties are augmented by further increases in network size, we take 7 of our $m=$ 0.0025 networks at $N=50$ and allow their evolution to continue, terminating the simulation when network growth became very slow. We then investigate the temporal evolution of network features by extracting the fittest network from 6 of our $28 m=0.0025$ evolutionary runs every 500 generations up to $N=50$, and then, in some cases, continuing the evolutionary runs until generation 4500 . To investigate how the properties of our evolved networks depend on the mutation load we run 8 independent randomly seeded evolutionary histories from $N=4$ to $N=50$ for $m=0.04,0.02,0.01$ and 0.005 respectively. Finally, we use our model to perform three specific investigations: first, we run 9 randomly seeded evolutionary histories without gene duplications; second, we investigate network plasticity (the ability of a network to maintain or increase fitness following rewiring mutations) by measuring a single evolved network's average robustness following 1000 independent single random edge additions; and third, we investigate the fitness-robustness properties of two $N \sim 50$ populations derived from running our model for each of the different mutation rates noted above.

All features shown in Table $\mathbf{3}$ are calculated from the fittest network extracted from the population at the end of each of 28 evolutionary runs at $N=50$. For the nine fitness measures, we take average values following 1000 repeated random mutations (of the particular type). For the dynamical stability investigations, we perform 1000 independent singlevertex random perturbations from random states on the networks' functional attractors, and determine the magnitudes of the respective subsequent avalanches.

\section{RESULTS}

We first investigate the characteristics of the fittest $N=$ 50 networks of each of our 28 populations that evolved at $m$

\footnotetext{
${ }^{5}$ For evolutionary systems, the approximate error threshold occurs when there is one mutation per organism per replication event. Higher mutation rates lead to irreversible information loss. For a primitive genome of 400 genes, the probability of mutation $p$ at the error threshold for $N$ embedded network modules' genes is given by $p=N / 400$. If $N=4, p=0.01$, and if $N=50, p=0.12$, as required.
}

Table 3. Network characteristics of the fittest network and their respective values calculated from 28 independent evolutionary simulations for $\boldsymbol{m}=\mathbf{0 . 0 0 2 5}$. See Fig. 2 for the distribution of values for features $2,5,6,7$, 8 and 9.

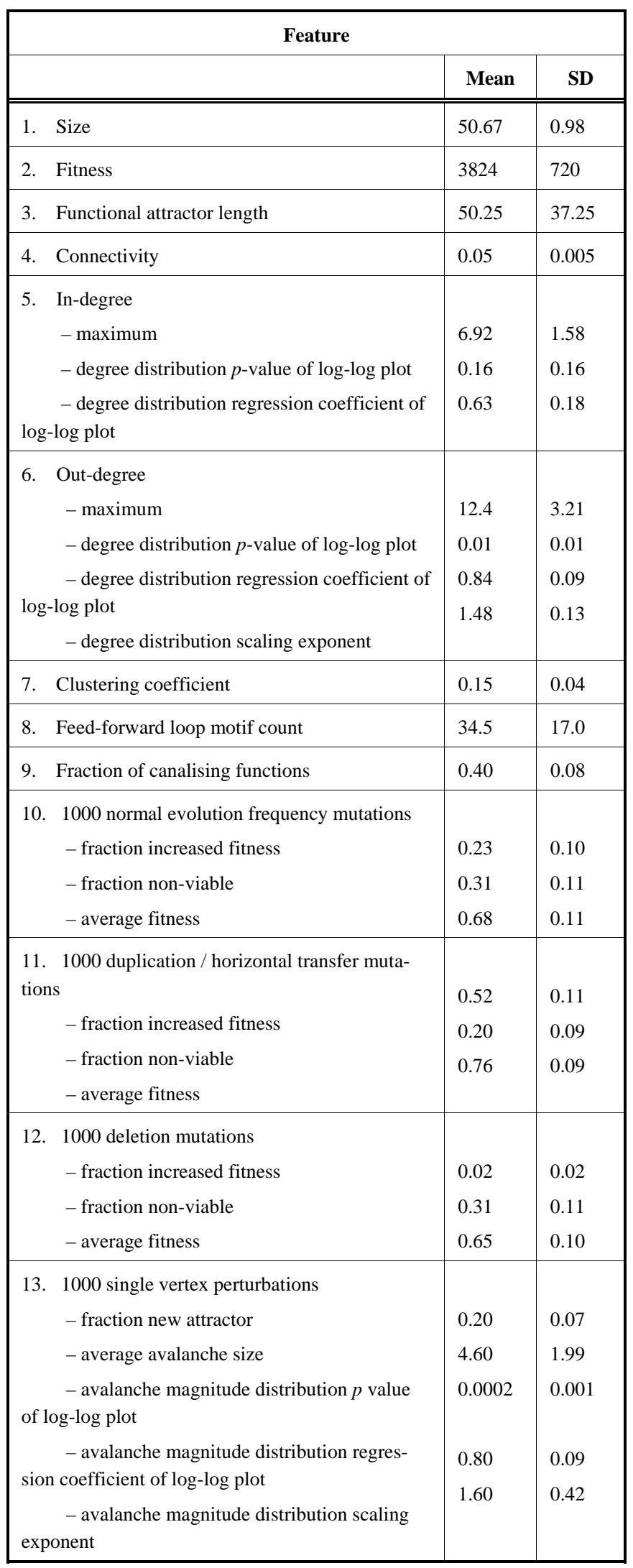



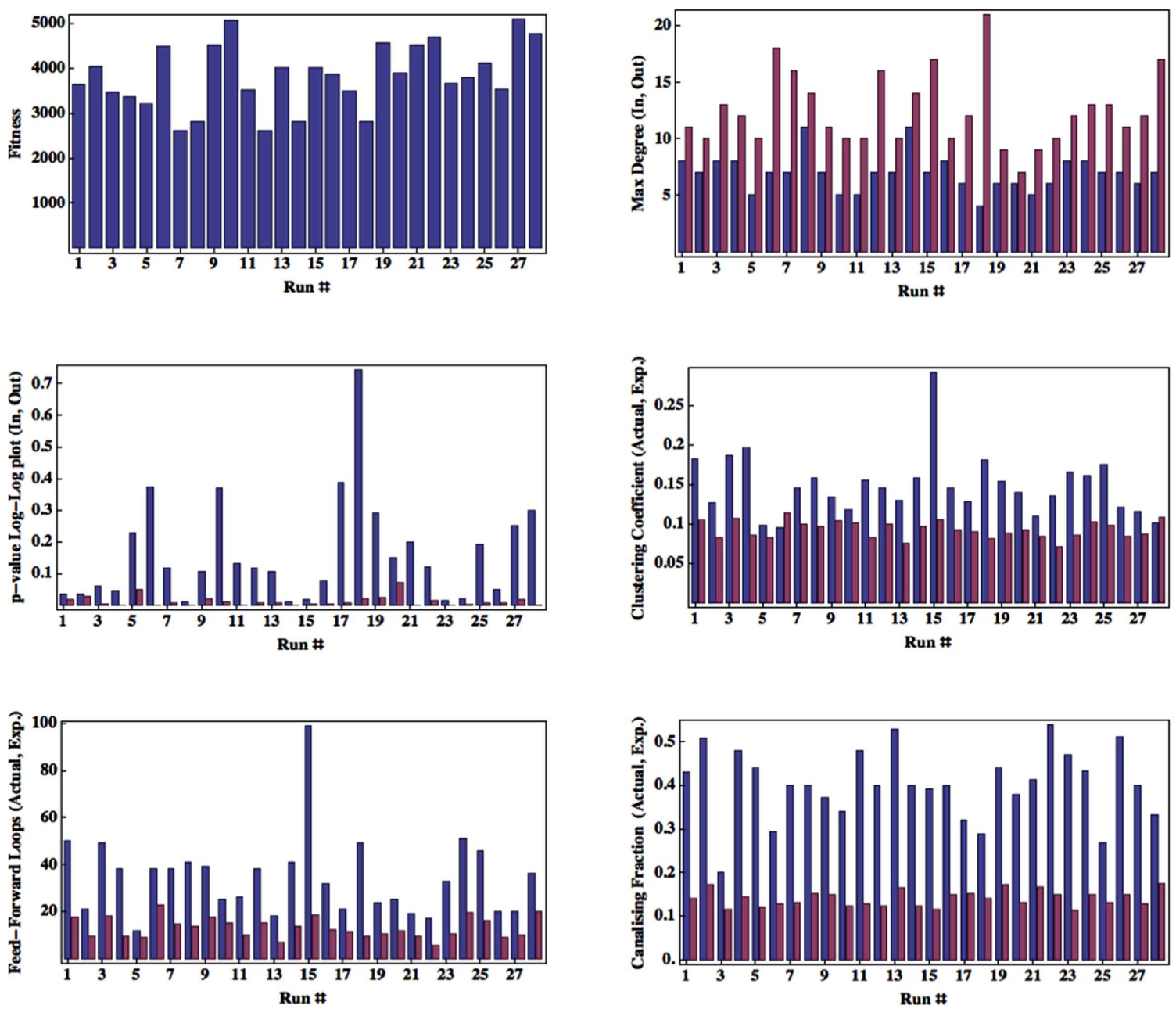

Fig. (3). Histogram showing distribution of feature values for the fittest member of 28 populations evolved at $m=0.0025$ where $N=$ 50. Fitness is shown in (a) while maximum in and out-degree are shown in (b). Note the maximum out-degree is always greater than the maximum in-degree. $p$ values (c) reveal that the straight line regression plot of log frequency versus log magnitude for the in-degree does not reach significance for any realisation; this result is in contrast to the out-degree distribution. Clustering coefficient (d) and number of feedforward loop motifs (e) are significantly greater than their corresponding values for random graphs of the same connectivity. The fraction of canalising Boolean inputs (f) is much higher than would be expected from the random generation of logic strings.

$=0.0025$ from $N=4$ until $N=50$; the results are shown in Table 3 and Fig. (3). Remarkably, all networks exhibit qualitatively similar architectural and dynamic features without parameter tuning or mutational bias: sparse connectivity, significant differences in the magnitudes of the in and outdegrees ( $p<10^{-8}$, Wilcoxon Rank Sum test (WRS), see Fig. 3b), scale-free out-degree distributions (with an average scaling exponent of 1.48, and a caveat that our use of the term scale-free pertains to our relatively limited range of measurement; average value of the linear regression log-log plot: $p=10^{-2}$; see Fig. 3c), exponentially decaying in-degree distributions, prominent clustering $(\langle C C\rangle=0.15$ versus $<C C>=0.09 \pm 0.01$ for the same number of randomly constructed networks with the same connectivity, $p<10^{-8}$, WRS, see Fig. 3d), increased prevalence of feed-forward loop motifs $(<F F L>=34.5$ versus $<F F L>=13.1 \pm 4.4$ for the same number of randomly constructed networks with the same connectivity, $p<10^{-8}$, WRS, see Fig. 3e), a high fraction of canalising input functions compared with randomly generated Boolean rules with the same length distribution ( $p$ $<10^{-9}$, WRS, see Fig. 3f), distributed robustness to mutation, and attractor dynamics that evolve to power-law avalanches of altered expression (with an average scaling exponent of 1.60 and an average $p$-value of the straight line regression of the log-log plot $<10^{-3}$ ) in response to single vertex perturbation - the hallmark of self-organised criticality.

We next investigate the possible relations between each of our 28 measured parameters (Table 3 ) among the 28 fittest networks by creating a 28 x 28 correlation matrix. We find one important result: a negative correlation between the networks' fraction of canalising functions and the magnitude of the networks' maximal out-degree (linear regression, $p=10^{-}$ $\left.{ }^{2}\right)$. We come back to this point in the Discussion.

We then take 7 of our $28 m=0.0025 N=50$ networks and let their evolution continue until network growth became very slow. Mean network size for these networks is 70 . We find similar features in these larger networks in comparison with our $N=50$ networks. Fig. (4) shows the key properties of the fittest network $(N=73)$ from one of these populations at generation 4500: scale-free out-degree distributions (linear regression log-log plot; $p<10^{-6}$ ) and self-organised critical dynamics (linear regression log-log plot; $p<10^{-15}$ ) are the two key signatures present in this network. Fig. (5) high- 
a

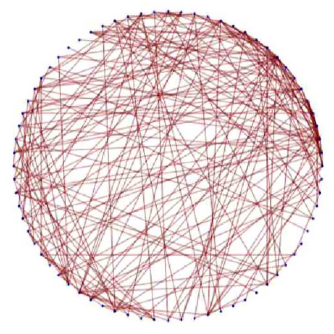

b

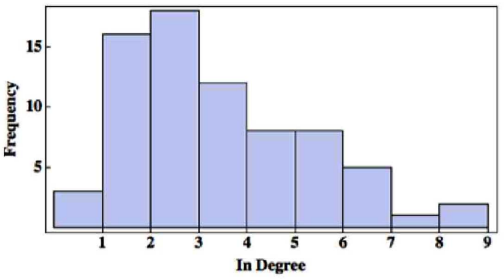

d

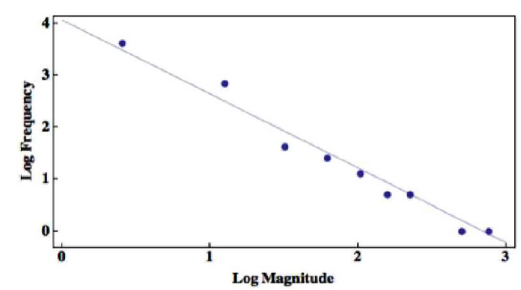

C

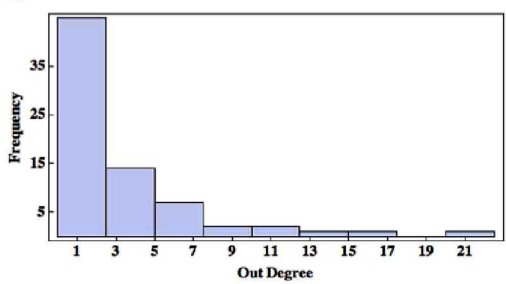

e

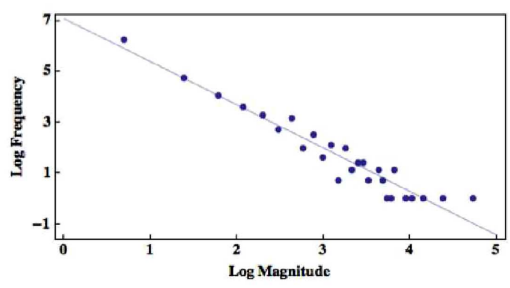

Fig. (4). Evolved characteristics for a $N=73$ network. Network graph (a) showing a highly connected region at top right. Histogram of in (b) and out (c) degree distributions reveal a fat tail for the out-degree distribution. This latter finding is reflected in (d), which shows a loglog plot of frequency versus outgoing connection counts. Over the scale of the plot, the relationship follows a straight line, with scaling exponent 1.33. This same relationship is evident in (e), where, following single vertex perturbation to random attractor states, the logfrequency of avalanche size is plotted against the log of avalanche size. Over the scale of measurement, the plot follows a straight line, indicating the dynamic behaviour of the network exhibits self-organised criticality. Here the scaling exponent is 1.71 .

a

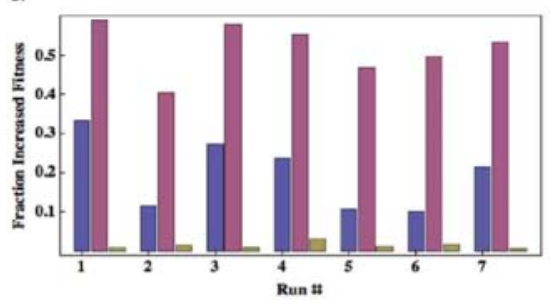

b

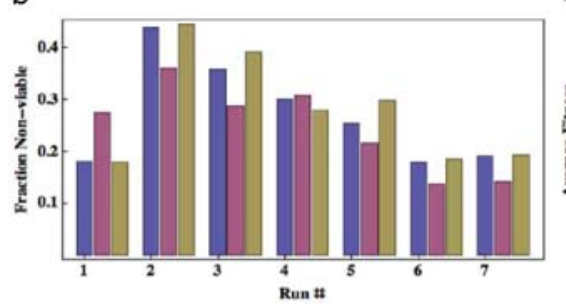

C

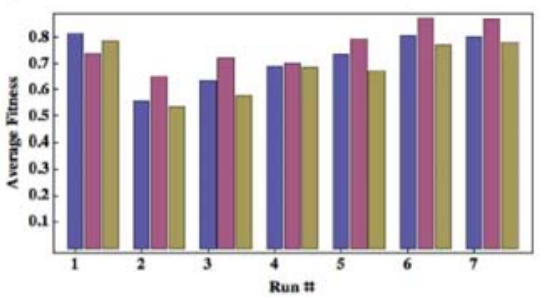

Fig. (5). Mutational robustness of networks for $\boldsymbol{N} \sim \mathbf{7 0}$. Here we investigate the robustness of the 7 fittest networks extracted from 7 independently evolved populations at $m=0.0025$. In (a), we represent evolvability by the fraction of 1000 random mutations that lead to increased fitness; in (b) we show the fraction of 1000 random mutations that lead to non-viability, while in (c) we show the average fitness of the network following 1000 random mutations. For each trio of columns, the first represents the frequency of mutation types as per the evolutionary run, while the second and third columns represent duplication / horizontal transfer and deletion mutations repectively.

lights the evolved robustness of these seven networks to mutations.

To investigate the temporal evolution of network features we sample the fittest member of $6 \mathrm{~m}=0.0025$ populations every 500 generations up to generation 4500; the important results are shown in Fig. (6). We note that while mutational robustness (the fraction of mutated networks that remain viable) increases slowly with time, significant robustness exists in small networks. Overall, evolvability (the fraction of mutated networks with increased fitness) remains unchanged as network size increases. Do any of the features found in Table $\mathbf{3}$ exhibit any dependence on the mutation load during evolution? We find that none of our network features are influenced by varying mutation rates with the exception of fitness; in this latter case there is a mild decrease in fitness for $N=50$ networks that evolve at mutation rates greater than baseline. Surprisingly, network robustness does not vary with mutation rate: evolvability, the non-viable fraction following mutations, and the average fitness following mutations does not change with mutation rate (data not shown).

We next perform three specific investigations: 9 evolutionary runs from $N=4$ to $N=50$ at $m=0.0025$ without gene duplications (in this case the duplication probabilities shown in Table 2 are replaced by horizontal transfer), an investigation of network plasticity, and a detailed investiga- 
tion of two $N=50$ populations that we recover from our simulations at each mutation load reported above. Fig. (7) reveals that scale-free out-degree distributions are not present in networks evolved without gene duplications (a comparison between maximal out-degree; $p<10^{-3}$, WRS). In addition, we find network clustering and the feed-forward loop motif count are much reduced in comparison to networks evolved with duplications ( $p<10^{-3}$, WRS); these results are similar to those expected from random graphs with the same connectivity. Fig. (7) also demonstrates an unex-
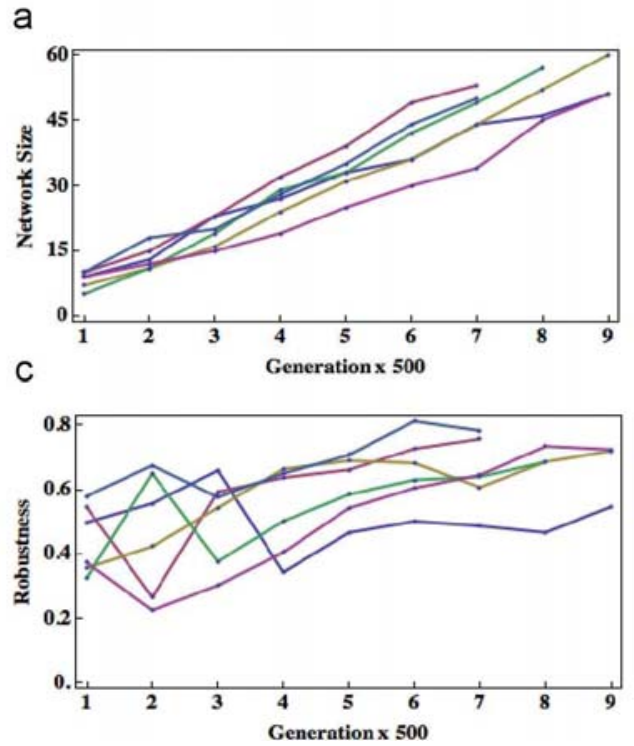

pected finding: networks evolved without duplications - and hence without scale-free architectures - exhibit greater mutational robustness ( $p<10^{-2}$, WRS), and contain a very high fraction of canalising functions $\left(p<10^{-2}\right.$, WRS). We find that despite these differences, networks evolved without duplications - like their evolved-with-duplication counterparts - also evolve to a self-organised critical state.

We now demonstrate the plasticity of our evolved networks. Following 1000 random and independent edge additions to a single large network, we find that while $19 \%$ mu-

b
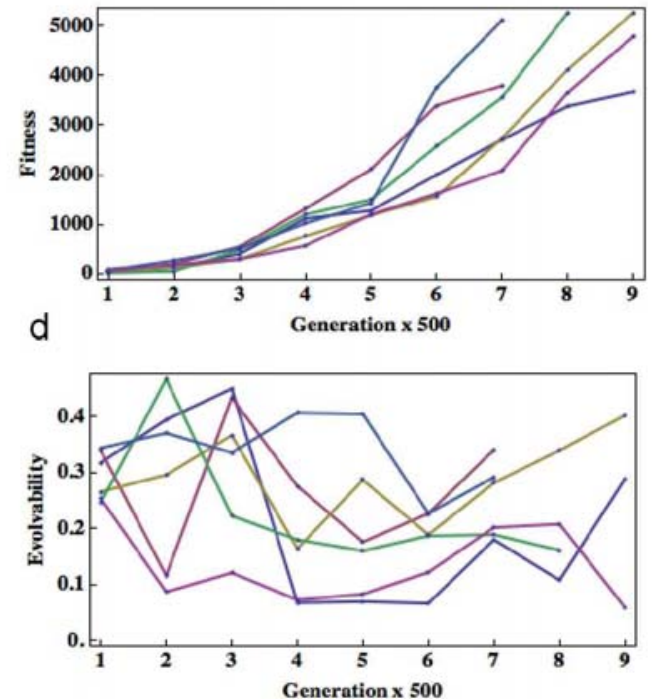

Fig. (6). Evolution of network features as a function of generation. Since our evolving populations contain 250 networks, each generation is equivalent to 250 Monte Carlo reproductive events, sampled from the population with a frequency proportional to fitness. Note fitness (a) grows faster than network size (b). Although robustness to mutation, on average, slowly increases with increasing complexity of the networks, one particular network was less robust compared with its much smaller ancestor. Evolvability does not increase during the evolution from very small to much larger networks.

a

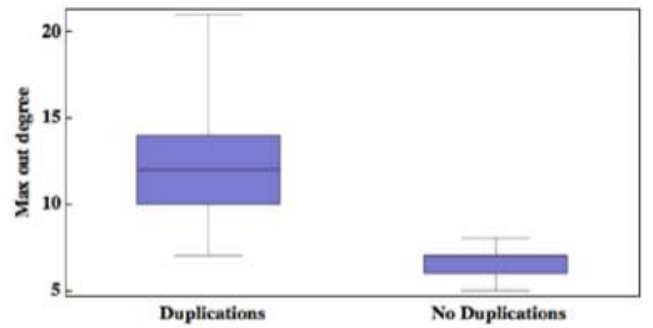

C

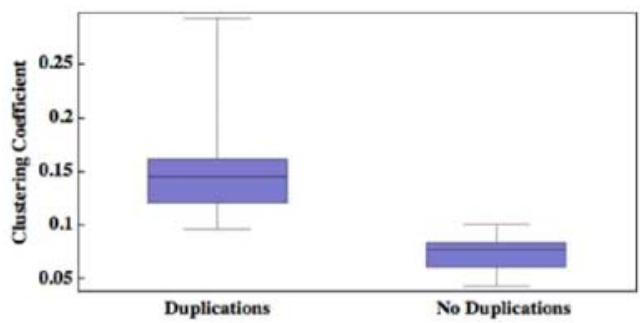

b

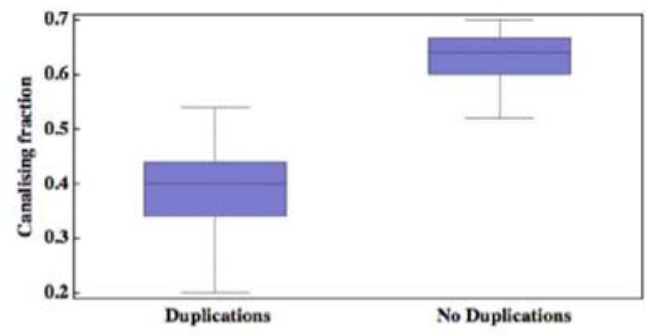

d

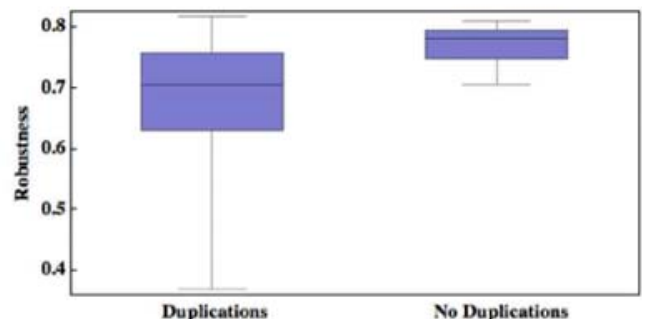

Fig. (7). Features of networks evolved with or without duplication events. Box-and-whisker plots demonstrating the differences between the two groups (duplications, $N=28$, and no duplications, $N=9$ ) with respect to maximal out-degree (a), canalising fraction (b), clustering coefficient (c) and mutational robustness (average fitness following 1000 random mutations, expressed as a fraction) (d). The differences between the two groups are all significant: $p<0.001$ (a-c); $p<0.003$ (d) (Wilcoxon rank sum test). 
tants are non-viable, the remainder are associated with an average fitness equal to $98 \%$ of the network's initial fitness. Moreover, $6 \%$ of mutants exhibit a gain in fitness. These results, which are strikingly similar to those obtained with $E$. coli [33], indicate that this network is highly evolvable.

Finally, we investigate the correlations between fitness and mutational or dynamic robustness of all members belonging to two $N=50$ populations evolved at each of the five mutation rates noted above. For nine populations, we find no evidence of intra-population correlations. One population demonstrates a correlation between fitness and mutational fragility; this result is shown in Fig. (8a) (linear regression, $p$ $<10^{-2}$ ). There is no evidence, however, that the distribution of members in any of our ten populations is evolving toward the efficient frontier, a hypothetical boundary in fitnessrobustness phase space [34].

\section{DISCUSSION}

Here we establish the validity of our model by demonstrating that the important large and small-scale architectural features of extant gene regulatory networks can be reproduced in an evolutionary setting that lacks any predetermined bias for the development of these features $[16,21,35]$. Our networks are evolvable, are robust to mutation, and their functional attractors are generally short (see Table 3), a result consistent with the observed simplicity of single-gene switching events in eukaryotes [36]. By reproducing the E-coli re-wiring experiment of Isalan et al., [33], we find similar quantitative results: our networks, like the genetic regulatory network in E-Coli, are plastic; that is, they can tolerate network re-wiring changes well, and will frequently benefit from such changes.

Our networks also exhibit self-organised criticality, a feature that evolves gradually with network growth, and is considered a general feature of cellular dynamics [20]. In the Boolean representation of genetic regulatory networks, although 'edge of chaos' dynamics are generally thought to require large frozen components [19,21], our networks are associated with attractor patterns where the majority of vertices oscillate. This contrary finding has important implications for the evolvability of networks [37]: homeostatic yet flexible dynamics can exist without functional redundancy. While it has been suggested that scale-free architectures may facilitate a reduction in functional redundancy [38], recent work suggests that criticality may maximise both robustness and evolvability [39]. Our results support all three of these contentions: first, since our fitness function implicitly selects for networks with low functional redundancy, we might expect all our networks to exhibit criticality: indeed, this is the case; and second and third, since our networks are critical even when small, we cannot refute the idea that this dynamic signature evolves readily in order to enhance (or, possibly, maximise) both robustness and evolvability.

\section{Real-World Fitness}

Does the success of our model in reproducing biologic network structure imply that within-network information processing is an important fitness measure in real biological systems? Although we have drawn from a large body of work to justify our fitness measure (see Methods), clearly the abstract nature of the mutual information content of gene expression patterns implies that it cannot, on its own, be the sole determinant of fitness. Details matter, and although we incorporate semantic details in our model by constraining the fidelity of reproduction, mutations in real biological systems may at times be associated with increased fitness and a reduction in functional complexity. One possible solution to this shortcoming would be to introduce an evolutionary algorithm where certain attractor patterns near to the current attractor $^{6}$ could be assigned a higher fitness, independent of the magnitude of the mutual information associated with the new attractor. However, giving greater fitness weightings to specific patterns (analogously to pattern-matching evolutionary algorithms) introduces teleology: evolution is constrained and becomes predictable, a less than desirable outcome. In any case, our overarching goal is not to gain insight with regard to real-world fitness measures; rather, we are motivated to develop a model that allows us to gain insight into the evolution of network architectures and dynamics. It is this latter consideration to which we now turn.

\section{The Origin of Large and Small-scale Network Structure}

While the origins and functions of extant biological gene regulatory network characteristics remain uncertain, there have been many investigations, both experimental and theoretical, that have attempted to address this issue, yielding a variety of explanations. In particular, the origins of scale-free out-degree distributions in biologic networks have been attributed, by various authors, to maximising information flow $[7,8]$, to facilitate mutational robustness $[6,40]$, as a consequence of neutral selection [9], and finally, as a by-product of gene duplications during evolution $[10,11,12]$. The latter explanation is still controversial; evidence obtained from the investigation of paralogous genes have also been used to argue that gene duplications are not responsible for the development of scale-free networks [41]. It is only the first explanation - maximising information flow within networks - that dovetails with the intuitive idea that selection effects shape biological phenotypes.

By demonstrating that networks grown without duplication mutations do not exhibit any of the typical architectural signatures found in extant genetic regulatory networks, we provide evidence that suggests duplications are an essential prerequisite for the evolutionary development of such features. Furthermore, the negative correlation between the maximal out-degree and the fraction of canalising inputs (demonstrable in our 28 networks grown from $N=4$ to $N=$ 50 (Fig. 8b) as well as in the comparison between networks grown with or without duplications (Fig. 7a, b) suggests - if we assume canalising inputs act to stabilise network dynamics at criticality - that scale-free architectures also contribute to the overall stability of the networks' dynamics. This result suggests that in biological systems, scale-free outdegree distributions are subject to positive selection. Indeed, the notion that positive selection for scale-free out-degree distributions may occur is augmented by a second line of evidence: when a hub regulates many genes, we may expect those regulated genes to regulate a number of common genes

\footnotetext{
${ }^{6}$ One method of quantifying the 'nearness' of two attractor patterns in a cellular automata is by calculating the Hamming distance.
} 
a

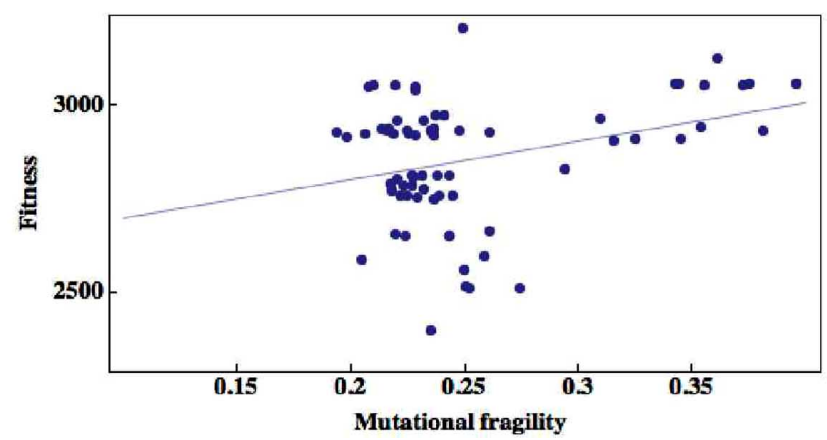

b

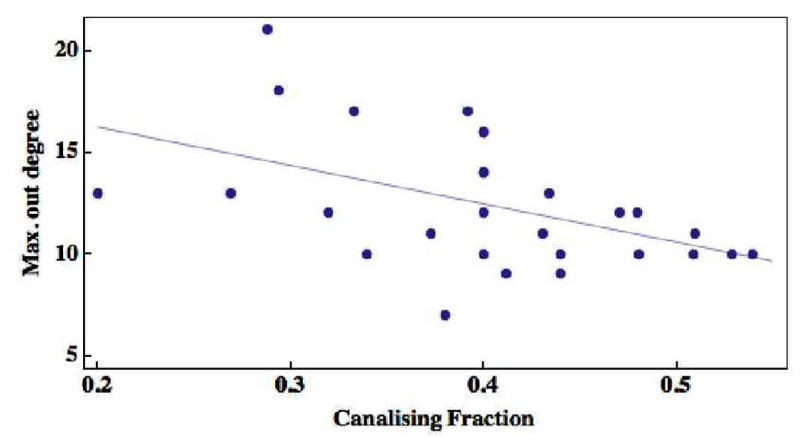

Fig. (8). Intra and inter-population correlations. Fitness-robustness phase space (a) for a population of $N=50$ networks evolved at $m=$ 0.0025. Each point represents identical members of the population. Note the correlation between mutational fragility and fitness, and the absense of evidence for the aggregation of points around the efficient frontier, a hypothetical boundary that would trace a line upward and to the right. For our 28 populations evolved at $m=0.0025$, there exists a negative correlation between the maximal out-degree and the canalising fraction (b). Results shown in Fig. 7a, b are strongly suggestive of a similar interpretation.

since it is likely the duplicated genes are those that the hubs regulate. However, when we examine our networks with respect to the genes regulated by the genes that are in turn regulated by the hubs, we find no evidence for gene commonality; indeed, all the second-tier regulated genes are equivalent to a cohort of genes selected at random. This result indicates that our evolved networks are subject to significant rewiring following duplication events, and implies the maintenance of hubs is an architectural feature subject to positive selection. While the authors of two papers advocating duplications as a cause of scale-free network architectures argue against positive selection, they do not rule out this possibility [11,12].

Perhaps surprisingly, our networks evolved without duplications - and therefore without scale-free out-degree distributions, prominent clustering or increased numbers of feed-forward loop motifs - perform just as well (or better) than networks evolved with duplications: in particular, mutational robustness is enhanced (Fig. 7d). The networks also exhibit self-organised criticality, indistinguishable from their scale-free counterparts. These results suggest evolution could have led to very different network structures, demonstrate that scale-free architectures are not a necessary prerequisite for the evolution of critical dynamics, and imply that gene duplications are an example of contingent mechanism. Our results allow us to draw some tentative biological conclusions: the molecular machinery that allows segments of DNA to be duplicated is not then a prerequisite for fully functioning networks to evolve, but since it occurs, it leads to certain architectural features - scale-free out-degree distributions, network clustering and increased numbers of feed-forward loop motifs - of which the former, and probably the latter two, are then subject to positive selection.

\section{The Evolution of Network Robustness and Evolvability}

In contrast to the paradigm that scale-free architectures confer mutational robustness to networks, we have found that scale-free architectures are less mutationally robust than their counterparts evolved without gene duplications. We expect this result is due to the higher canalising fraction in the networks evolved without duplications, potentially augmented by the susceptibility of networks with hub genes to be subject to catastrophic failure. We conclude that there is a significant trade-off between the maximal out-degree and the fraction of canalising inputs with respect to the mutational robustness of evolved networks, a result that suggests canalising inputs do not just stabilise global dynamics, but play a significant role in conferring mutational robustness. The interplay between network architecture and logic implies that the properties of network architectures, if examined in isolation, may yield incorrect conclusions with regard to their functional significance.

Is robustness a key driver for the evolution of complex features? While a previously reported conclusion based on the results of a fitness-free evolutionary model suggests that selection for robustness may play a non-trivial role in shaping network architecture and dynamics [21], other authors have suggested selection for robustness may facilitate network growth $[42,43]$. Selection for robustness is likely to play an important role in the evolution of the phage lambda genetic regulatory circuit [44], and may be analogous to 'survival of the flattest' [45]. Many of the above findings are perhaps not surprising. After all, robustness is, in a sense, the ultimate basis for fitness. We might think of robustness as the stage on which the ballet of neutral and fitness-driven evolution is played out. Many fundamental properties of gene network architecture and logic are likely to be determined by an absolute requirement for mutational robustness. But the evolutionary relationships between network features that confer robustness on one hand, and fitness on the other, are less clear. If robustness were a key driver of the evolution of the complex features that in turn augment network fitness then we would expect that, within evolving populations, a positive correlation between fitness and robustness should exist, and very few, if any, members of populations with both low fitness and low robustness should be present. On the other hand, if robustness drives the evolution of complex features that are deleterious to fitness, we would expect a within-population trade-off between fitness and robustness. This latter possibility is analogous to the idea of an efficient frontier, noted previously and introduced by Kitano, where such a trade-off defines a boundary in fitness-robustness phase space that members of populations cannot cross [34]. 
Yet there exists a third possibility: complex features conferring robustness to networks may only have marginal effects on fitness - they are essentially neutral. What do our results suggest? First, with the exception of one population evolved at $m=0.0025$ (Fig. 8a), none of our other nine intrapopulation fitness-robustness scatter plots provide any evidence for fitness-robustness correlations, or evidence that the populations are evolving toward the efficient frontier. Second, we cannot demonstrate any inter-population correlations between fitness and robustness when we examine our 28 independent simulations at $m=0.0025$. Finally, we have shown that significant robustness is already present in small networks (Fig. 6c), and that evolvability does not increase with increasing network complexity (Fig. 6d). Taken together, this line of evidence suggests robustness and evolvability are not major drivers for the evolution of the complex features associated with either fitness gains or losses. Our results thus support the neutral hypothesis, Fig. 8a notwithstanding. It is important to note that the term 'complex feature' is not precise; in this regard we suggest the existence of robustness and evolvability in networks may be due to subtle large or small-scale architectural organisation - complex features, nonetheless - that only have marginal effects on fitness and hitherto remain unidentified.

\section{CONFLICT OF INTEREST}

None declared.

\section{ACKNOWLEDGEMENTS}

None declared.

\section{REFERENCES}

[1] Pigliucci, M. Do we need an extended evolutionary synthesis? Evolution, 2007, 61: 2743-2749.

[2] Carroll, R.L. Towards a new evolutionary synthesis. Trends. Ecol. Evol., 2000, 15: 27-32.

[3] Avise, J.C.; Ayala, F.J. In the light of evolution I: Adaptation and complex design. Proc. Natl. Acad. Sci. USA, 2007, 104 (Suppl 1), 8563-8566.

[4] Koentges, G. Evolution of anatomy and gene control. Nature, 2008, 451, 658-663.

[5] Poelwijk, F.J.; Kiviet, D.J.; Weinreich, D.M.; Tans, S.J. Empirical fitness landscapes reveal accessible evolutionary paths. Nature, 2007, e 445, 383-386.

[6] Barabasi, A.L.; Albert, R. Emergence of scaling in random networks. Science, 1999, 286, 509-512.

[7] Braha, D.; Bar-Yam, Y. Topology of large-scale engineering problem-solving networks. Phys. Rev. Stat. Nonlin. Soft-Matter.Phys. 2004, 69, 016113, 1-7.

[8] Sole, R.V.; Valverde, S. Information theory of complex networks: On evolution and architectural constraints. Santa Fe Institute Working Paper, 03-11-061.

[9] Lynch, M. The evolution of genetic networks by non-adaptive processes. Nat. Rev. Genet., 2007, 8: 803-813.

[10] Gelfand, M.S. Evolution of transcriptional regulatory networks in microbial genomes. Curr. Opin. Struct. Biol., 2006, 16: 420-429.

[11] Amoutzias, G.R.; Robertson, D.L.; Oliver, S.G.; Bornberg-Bauer, E. Convergent evolution of gene networks by single gene duplications in higher eukaryotes. EMBO Rep., 2004, 5 (3), 274-279.

[12] van Noort, V,; Snel, B.; Huynen, M. The yeast co-expression network has a small-world scale free architecture and can be explained by a simple model. EMBO Rep., 2004, 5 (3), 280-284.

[13] Vazquez, A.; Rammini, A.; Maritan, A.; Vespignany, A. Modeling of protein interaction networks. Complexus, 2003, 1: 38-44.
[14] Foster, D.V.; Kauffman, S.A. Network growth models and genetic regulatory networks. Phys. Rev. Stat. Nonlin. Soft-Matter.Phys., 2006, 73: 031912, 1-8.

[15] Babu, M.M.; Teichmann, S.A.; Aravind, L. Evolutionary dynamics of prokaryotic transcriptional regulatory networks. J. Mol. Biol., 2006, 358: 614-633.

[16] Babu, M.M. Structure, evolution and dynamics of transcriptional regulatory networks. Biochem. Soc. Trans., 2010, 38: 1155-1178.

[17] Cordero, O.; Hogeweg, P. Feed-forward loop circuits as a sideeffect of genome evolution. Mol. Biol. Evol., 2006, 23(10): 19311936.

[18] Harris, S.E.; Sawhill, B.K.; Wuensche, A.; Kauffman, S. A model of transcriptional regulatory networks based on biases in the observed regulation rules. Complexity, 2002, 7 (4): 23-32.

[19] Kauffman, S.A. The Origins of Order. Self-organization and selection in evolution. Oxford University Press: New York, USA, Oxford, UK, 2003.

[20] Ramo, P.; Kesseli, J.; Yli-Harja, O. Perturbation avalanches and criticality in gene regulatory networks. J. Theor. Biol., 2006, 242,: 164-170.

[21] Bornholdt, S. Modelling genetic networks and their evolution: A complex dynamical systems perspective. Biol. Chem., 2001, 382: 1289-1299.

[22] Lenski, R.E.; Ofria, C.; Pennock, R.T.; Adami, C. The evolutionary origin of complex features. Nature, 2003, 423: 139-144.

[23] Carroll, S.B. Endless forms most beautiful. Phoenix: London, UK, 2005.

[24] Ay, N.; Olbrich, E.; Bertschinger, N.; Jost, J. A unifying framework for complexity measures of finite systems. Santa Fe Institute Working Paper, 06-08-028.

[25] Komili, S.; Silver, P.A. Coupling and co-ordination in gene expression processes: a systems biology view. Nat. Rev. Genet., 2008, 9: 38-48.

[26] Arendt, D. A kernel bears fruit. Science, 2006, 314: 1085-1086.

[27] Lozada-Chavez, I.; Janga, S.C.; Collado-Vides, J. Bacterial regulatory networks are extremely flexible in evolution. Nucleic. Acids. Res., 2006, 34: 3434-3445.

[28] Wagner, A. Does evolutionary plasticity evolve? Evolution, 1996, 50: 1008-1023.

[29] Quayle, A.P.; Bullock, S. Modelling the evolution of genetic regulatory networks. J. Theor. Biol., 2006, 238: 737-753.

[30] Crombach, A.; Hogeweg, P. Evolution of evolvability in gene regulatory networks. PLoS Comp. Biol., 2008, 4: e1000112.

[31] Adami, C. Introduction to artificial life. Springer-Verlag: Berlin, 1998.

[32] Mushegian, A.R.; Koonin, E.V. A minimal gene set for cellular life derived by comparison of complete bacterial genomes. Proc. Natl. Acad. Sci. USA, 1996, 93: 10268-10273.

[33] Isalan, M., et al. Evolvability and hierarchy in rewired bacterial gene networks. Nature, 2008, 452: 840-845.

[34] Kitano, H. Violations of robustness trade-offs. Mol. Syst. Biol., 2010, 6: 384.

[35] Alon, U. An introduction to systems biology: Design principles of biological circuits. Chapman Hall / CRC: Boca Raton, Florida, USA, 2007.

[36] Cho, R.J.; Campbell, M.J.; Winzeler, E.A.; Steinmetz, L.; Conway, A.; Wodicka, L.; Wolfsberg, T. G.; Gabrielian, A. E.; Landsman, D.; Lockhart, D. J.; Davis, R.W. A genome-wide transcriptional analysis of the mitotic cell cycle. Mol. Cell, 1998, 2: 65-73.

[37] Pigliucci, M. Is evolvability evolvable? Nat. Rev. Genet., 2008, 9: 75-82.

[38] Fortuna, M.A.; Melian, C.J. Do scale-free regulatory networks allow more expression than random ones? J. Theor. Biol., 2007, 247: 331-336.

[39] Aldana, M.; Balleza, E.; Kauffman, S.; Resendiz, O. Robustness and evolvability in genetic regulatory networks. J. Theor. Biol., 2007, 245: 433-448.

[40] Wagner, A. Robustness against mutations in genetic networks of yeast. Nat. Genet., 2000, 24: 355-361.

[41] Teichmann, S.A.; Babu, M.M. Genetic network growth by duplication. Nat. Genet., 2004, 5: 492-496.

[42] Csete, M.E.; Doyle, J.C. Reverse engineering of biological complexity. Science, 2002, 295: 1664-1669.

[43] Soyer, O.S.; Bonhoeffer, S. Evolution of complexity in signalling pathways. Proc. Natl. Acad. Sci. USA, 2006, 103 (44), 1633716342. 
[44] Little, J.W.; Shepley, D.P.; Wert, D.P. Robustness of a gene regulatory circuit. EMBO J, 1999, 18 (15),: 4299-4307.
[45] Wilke, C.O.; Wang, J.L.; Ofria, C.; Lenski, R.E.; Adami, C. Evolution of digital organisms at high mutation rates leads to survival of the flattest. Nature, 2001, 412: 331-333.

Received: November 17, 2011

(C) Gilmore and Green.; Licensee Bentham Open.

This is an open access article licensed under the terms of the Creative Commons Attribution Non-Commercial License (http://creativecommons.org/licenses/by-nc/3.0/) which permits unrestricted, non-commercial use, distribution and reproduction in any medium, provided the work is properly cited. 